\title{
Loop-deformed Poincaré algebra
}

\author{
Jakub Mielczarek \\ Institute of Physics, Jagiellonian University, Reymonta 4, 30-059 Cracow, Poland \\ Department of Fundamental Research, National Centre for Nuclear Research, \\ Hoża 69, 00-681 Warsaw, Polandt
}

\begin{abstract}
In this essay we present evidence suggesting that loop quantum gravity leads to deformation of the local Poincare algebra within the limit of high energies. This deformation is a consequence of quantum modification of effective off-shell hypersurface deformation algebra. Surprisingly, the form of deformation suggests that the signature of space-time changes from Lorentzian to Euclidean at large curvatures. We construct particular realization of the loop-deformed Poincaré algebra and find that it can be related to curved momentum space, which indicates the relationship with recently introduced notion of relative locality. The presented findings open a new way of testing loop quantum gravity effects.
\end{abstract}

\footnotetext{
a Essay written for the Gravity Research Foundation 2013 Awards for Essays on Gravitation. Submitted on 30 March 2013.

† jakub.mielczarek@uj.edu.pl
}

Typeset by REVTEX 
In crystals, symmetries of continuous rotations and translations are broken due to discrete nature of the molecular lattice structure. Similarly, it is usually expected that isometries of Minkowski space, described by the Poincaré group, are broken or deformed due to granularity of space at the Planck scale. Nevertheless, one can imagine that some types of discontinuity may preserve the large scale symmetries.

Let us see what it looks like in loop quantum gravity (LQG) [1], which is a background independent approach to quantize gravity. In LQG, "atomic" nature of space is reflected by discrete spectra of geometrical operators, such as area and volume. However, this feature alone does not guarantee violation of Lorentzian symmetry. Similarly, a discrete spectrum of square of the angular momenta operator does not imply breaking of the rotational invariance. Indeed, as pointed out by Rovelli and Speziale [2], quantum amplitudes of LQG are manifestly Lorentz covariant. Their result concerns kinematical sector of the theory comparably to discreteness of spectra of the geometrical operators. However, LQG is not only kinematics but also constraints that must be imposed in order to extract physical states. Furthermore, the off-shell algebra of these constraints carries information about symmetries of the theory.

At the classical level, this algebra corresponds to hypersurface deformation algebra, encoding general covariance, a cornerstone of general relativity (GR). However, the fate of this algebra at the quantum level is unknown due to the complicated form of the quantum constraints. Nevertheless, significant progress has recently been made in analysis of the effective off-shell algebra, where LQG effects were introduced through systematic modifications of the classical constraints. This analysis was performed for both, spherically symmetric configurations [3, 4] and perturbative inhomogeneities on the flat FriedmannRobertson-Walker (FRW) background [5, 6]. Main message coming from these studies is that the algebra of effective constraints is deformed with respect to its classical counterpart. Namely, it takes the following form:

$$
\begin{aligned}
& \left\{D\left[M^{a}\right], D\left[N^{a}\right]\right\}=D\left[M^{b} \partial_{b} N^{a}-N^{b} \partial_{b} M^{a}\right], \\
& \left\{D\left[M^{a}\right], S^{Q}[N]\right\}=S^{Q}\left[M^{a} \partial_{b} N-N \partial_{a} M^{a}\right], \\
& \left\{S^{Q}[M], S^{Q}[N]\right\}=\beta D\left[q^{a b}\left(M \partial_{b} N-N \partial_{b} M\right)\right],
\end{aligned}
$$


where $\beta$ is a deformation factor, equal one in the classical theory with Lorentzian signature. Superscript $Q$ indicates that scalar constraint $S$ is quantum corrected, whereas diffeomorphism constraint $D$, in the spirit of LQG, holds the classical form.

Functional form of the factor $\beta$ depends on whether the so-called inverse volume or holonomy quantum corrections are applied. For inverse volume corrections, $\beta$ depends on spatial metric $q_{a b}$, while holonomy corrections introduce dependence on extrinsic curvature. Interestingly, for both types of corrections, $\beta$ falls to zero within the regime of strong quantum gravitational effects. In consequence, the algebra of constraints becomes reduced to the ultralocal form [7] which prohibits any causal contacts between space points. Because information (waves) cannot propagate spatially, this phase of gravity is also known as asymptotic silence [8]. What is extremely interesting, is that such fancy behavior is expected also in the high curvature limit of GR. This phenomenon is known as Belinsky-Khalatnikov-Lifshitz (BKL) conjecture [9, 10].

However, that is not all. In case of holonomy corrections, one can cross the ultralocal stage and go to regime of negative $\beta$. Before we interpret what it means let us firstly say a few words on the origin of holonomy corrections. To be more specific, let us focus on example of FRW cosmology. In this case, holonomy corrections are introduced formally by replacing extrinsic curvature $\bar{k}$ by $\sin (\delta \bar{k}) / \delta$ function, with $\delta$ being a function of a scale factor. Because the $\delta \bar{k} \rightarrow \delta \bar{k}+2 \pi$ symmetry is introduced, the procedure can be seen as periodification of the real line to $U(1)$. The classical limit is recovered for $\delta \bar{k} \ll 1$

It was shown that for both, spherically symmetric models and cosmological perturbations with holonomy corrections, the deformation factor $\beta$ takes the same cosine form. Additionally, in case of cosmological perturbations, we can express $\beta$ in terms of matter energy density $\rho$ [6]:

$$
\beta=\cos (2 \bar{\mu} \gamma \bar{k})=1-2 \frac{\rho}{\rho_{\mathrm{c}}} \in[-1,1]
$$

where maximal energy density $\rho_{\mathrm{c}}=\frac{3}{8 \pi G \lambda^{2} \gamma^{2}} \sim \rho_{\mathrm{Pl}}$ and the Planck energy density $\rho_{\mathrm{Pl}} \equiv E_{\mathrm{Pl}}^{4}$, with $E_{\mathrm{Pl}} \approx 1.22 \cdot 10^{19} \mathrm{GeV}$. It is clear that at low energy densities $\left(\rho \ll \rho_{\mathrm{c}}\right)$ the classical limit $(\beta=1)$ is recovered. However, while passing to regime of high energy densities, deviations from the classical theory are evident. Firstly, while approaching $\rho=\rho_{\mathrm{c}} / 2$ we 
"slow down" to the state of asymptotic silence. Secondly, for $\rho<\rho_{\mathrm{c}} / 2$, sign of $\beta$ reverses, which is associated with change of the metric signature from Lorentzian to Euclidean [11]. Amazingly, the possibility of Euclidezation of space-time due to quantum gravitational effects was anticipated already by Hartle and Hawking in their famous no-boundary proposal [12].

What we have learned so far is that the effective algebra of constraints is deformed due to loop quantum gravity effects in a very interesting way. Consequently, general covariance is deformed and because Lorentz transformations and translations are special types of coordinate transformations, we expect that algebra of generators of these transformations is deformed as well. The crucial observation made by Bojowald and Paily was that this algebra can be recovered from the hypersurface deformation algebra by considering linear laps function $N$ and the shift vector $N^{a}[13]$ :

$$
N(x)=\Delta t+v_{a} x^{a}, \quad N^{a}(x)=\Delta x^{a}+R_{b}^{a} x^{b},
$$

together with the flat spatial metric $q_{a b}=\delta_{a b}$. Taking this into account, they showed that the resulting deformation of the Poincare algebra is a special case of a broader class of deformations, with modifications of generators of boosts, studied in Ref. [14]. However, as we show here, modification of boosts is not required if $\beta$ depends on generator of time translation only. In that case, the loop-deformed Poincaré algebra can be obtained from the following Heisenberg algebra

$$
\left[X_{\mu}, X_{\nu}\right]=0, \quad\left[P_{\mu}, P_{\nu}\right]=0, \quad\left[X_{\mu}, P_{\nu}\right]=i g_{\mu \nu}\left(P_{0}\right)
$$

where $g_{\mu \nu}\left(P_{0}\right)=\operatorname{diag}\left(-\beta\left(P_{0}\right), 1,1,1\right)$. By introducing classical generators of rotations $J_{i} \equiv \frac{1}{2} \epsilon_{i j k}\left(X_{j} P_{k}-X_{k} P_{j}\right)$ and boosts $K_{i} \equiv X_{i} P_{0}-X_{0} P_{i}$, we find that the following algebra is fulfilled:

$$
\begin{aligned}
{\left[J_{i}, J_{j}\right] } & =i \epsilon_{i j k} J_{k}, \quad\left[J_{i}, K_{j}\right]=i \epsilon_{i j k} K_{k}, \quad\left[K_{i}, K_{j}\right]=-i \beta\left(P_{0}\right) \epsilon_{i j k} J_{k}, \\
{\left[J_{i}, P_{j}\right] } & =i \epsilon_{i j k} P_{k}, \quad\left[K_{i}, P_{j}\right]=i \delta_{i j} P_{0}, \quad\left[J_{i}, P_{0}\right]=0, \\
{\left[K_{i}, P_{0}\right] } & =i \beta\left(P_{0}\right) P_{i}, \quad\left[P_{i}, P_{j}\right]=0, \quad\left[P_{i}, P_{0}\right]=0 .
\end{aligned}
$$


This algebra has the same form as the one found in Ref. [13]. However, here the boosts are classical and $\beta$ depends on $P_{0}$ only. The classical Poincaré algebra is recovered for $\beta \rightarrow 1$, while for $\beta=0$ we obtain Carrollian limit [15] describing ultralocal space-time. Furthermore, for $\beta=-1$, the algebra describes isometries of the 4D Euclidean space. Therefore, we have full compatibility with the conclusions derived at the level of algebra of constraints.

It is worth stressing that, while the loop-deformed Poincaré algebra is recovered from the algebra of constraints in the limit of flat space-time, the metric entering Heisenberg algebra reveals explicit dependence on $P_{0}$. This can be associated with curvature of momentum space, which suggests relation of our approach with recently introduced concept of relative locality [16, 17]. An unavoidable consequence of the momentum space curvature is modification of dispersion relation of particles, e.g. photons.

Because the first Casimir invariant for our loop-deformed Poincaré is

$$
\mathcal{C}_{1}=-\int_{0}^{P_{0}} \frac{2 y}{\beta(y)} d y+P_{i}^{2}
$$

the dispersion relation of photon takes the form $\int_{0}^{E} \frac{2 y}{\beta(y)} d y=\mathbf{p}^{2}$, where we defined $E=P_{0}$ and $\mathbf{p}^{2}=\left(P_{i}\right)^{2}$. For $\beta=1$, the classical relation $E^{2}=\mathbf{p}^{2}$ is correctly recovered. By differentiating the deformed dispersion relation with respect to $E$ one can show that $v_{\mathrm{gr}} v_{\mathrm{ph}}=\beta$, where $v_{\mathrm{gr}}$ and $v_{\mathrm{ph}}$ are group and phase velocities respectively. These velocities are no longer constant but vary as a function of energy. Furthermore, under the following assumptions: $0<\beta \leq 1, \beta(0)=1$ and $\frac{d \beta}{d E} \leq 1$, one can prove that they never exceed the speed of flight in vacuum $\left(v_{\mathrm{ph}} \leq 1, v_{\mathrm{gr}} \leq 1\right)$.

As an example, let us take $\beta(E)=1-\frac{E^{2}}{E_{*}^{2}}$. However, for other choices, e.g. $\beta(E)=$ $\cos \left(E / E_{*}\right)$, results are qualitatively the same. The corresponding energy dependence of the group velocity, given by formula

$$
v_{\mathrm{gr}}=\left(1-\frac{E^{2}}{E_{*}^{2}}\right) \frac{E_{*}}{E} \sqrt{-\log \left(1-\frac{E^{2}}{E_{*}^{2}}\right)}=1-\frac{3}{4}\left(\frac{E}{E_{*}}\right)^{2}+\mathcal{O}\left(E^{4}\right),
$$

is shown in Fig. 1, The free parameter $E_{*}$ can be constrained by studying time lags of high energy photons arriving from the distant astrophysical sources, such as gamma ray 


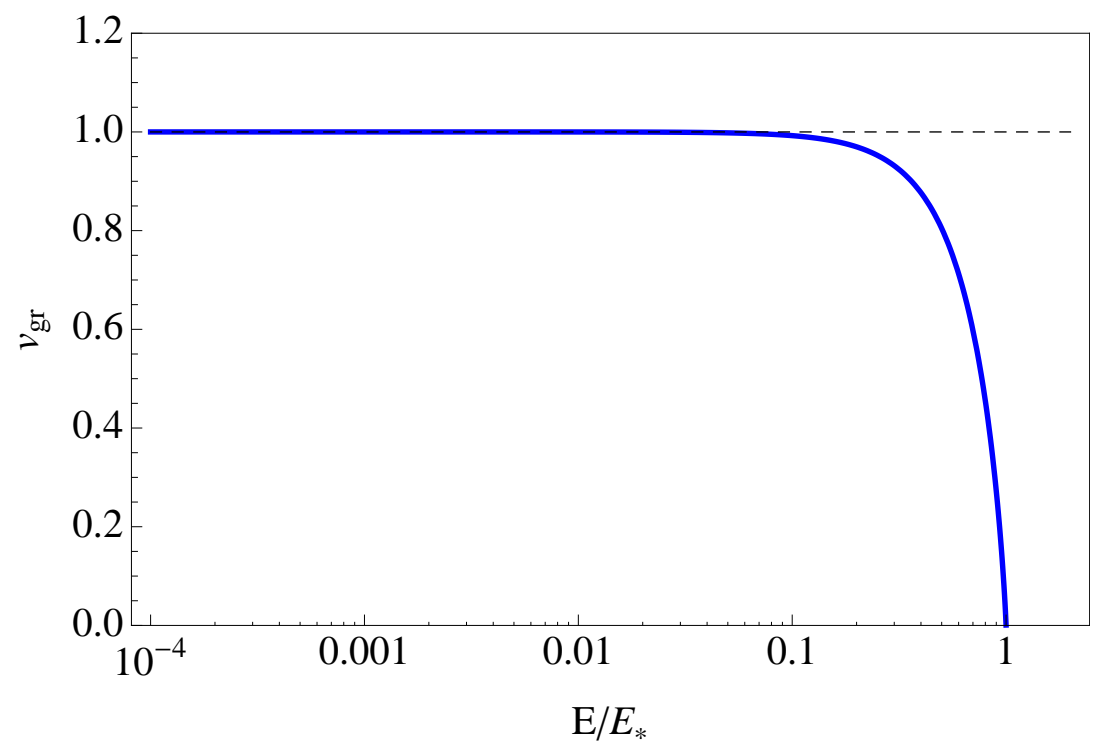

FIG. 1. Group velocity of photons is represented here as a function of energy for the model with deformation function $\beta(E)=1-\frac{E^{2}}{E_{*}^{2}}$. The group velocity falls to zero while $E \rightarrow E_{*}$, which corresponds to the Carrollian limit $(\beta \rightarrow 0)$.

bursts (GRB) [18]. In our case, this time lag is expressed as $\tau \simeq \frac{L}{c} \frac{3}{4}\left(\frac{E}{E_{*}}\right)^{2}$, where $L$ is a distance to source. Using constraint from the GRB 090510 detected by the Fermi satellite [19] we find that $E_{*}>4.7 \cdot 10^{10} \mathrm{GeV}$. The obtained constraint is weak relative to the Planck scale because quantum corrections are quadratic here, as suggested by the cosine form of $\beta$. Nevertheless, a new possibility of testing loop quantum gravity emerges. What is especially worth stressing is that both, dynamics of very early universe and propagation of astrophysical particles are affected by the same deformation $\beta$. This provides a unique possibility of constraining the same physics by using completely different observations. This might open a new stage in cosmic search for the quantum gravity effects.

[1] A. Ashtekar, J. Lewandowski and , Class. Quant. Grav. 21 (2004) R53 [gr-qc/0404018].

[2] C. Rovelli and S. Speziale, Phys. Rev. D 83 (2011) 104029 [arXiv:1012.1739 [gr-qc]].

[3] J. D. Reyes, PhD thesis, The Pennsylvania State University, 2009 
[4] M. Bojowald, G. M. Paily and , Phys. Rev. D 86 (2012) 104018 [arXiv:1112.1899 [gr-qc]].

[5] M. Bojowald, G. M. Hossain, M. Kagan, S. Shankaranarayanan and, Phys. Rev. D 78 (2008) 063547 [arXiv:0806.3929 [gr-qc]].

[6] T. Cailleteau, J. Mielczarek, A. Barrau and J. Grain, Class. Quant. Grav. 29 (2012) 095010 [arXiv:1111.3535 [gr-qc]].

[7] C. J. Isham, Proc. Roy. Soc. Lond. A 351 (1976) 209.

[8] J. Mielczarek, AIP Conf. Proc. 1514 (2012) 81 [arXiv:1212.3527][gr-qc]].

[9] V. A. Belinskii, I. M. Khalatnikov and E. M. Lifshitz, Adv. Phys. 19 (1970) 525; 31 (1982) 639.

[10] L. Andersson, H. van Elst, W. C. Lim, C. Uggla, Phys. Rev. Lett. 94 (2005) 051101 [gr-qc/0402051].

[11] J. Mielczarek, arXiv:1207.4657 [gr-qc].

[12] J. B. Hartle and S. W. Hawking, Phys. Rev. D 28 (1983) 2960.

[13] M. Bojowald and G. M. Paily, arXiv:1212.4773 [gr-qc].

[14] D. Kovacevic, S. Meljanac, A. Pachol and R. Strajn, Phys. Lett. B 711 (2012) 122 [arXiv:1202.3305 [hep-th]].

[15] J-M. Levy-Leblond, Annales de l'I.H.P., section A, tome 3, No 1 (1965).

[16] G. Amelino-Camelia, L. Freidel, J. Kowalski-Glikman and L. Smolin, Phys. Rev. D 84 (2011) 084010.

[17] G. Amelino-Camelia, L. Freidel, J. Kowalski-Glikman and L. Smolin, Gen. Rel. Grav. 43 (2011) 2547 [Int. J. Mod. Phys. D 20 (2011) 2867].

[18] G. Amelino-Camelia, J. R. Ellis, N. E. Mavromatos, D. V. Nanopoulos, S. Sarkar and, Nature 393 (1998) 763 [astro-ph/9712103].

[19] M. Ackermann et al. [Fermi GBM/LAT Collaboration], Nature 462 (2009) 331 [arXiv:0908.1832 [astro-ph.HE]]. 